\title{
Neuroanatomical Correlates of Perceived Usability
}

\author{
Chi Thanh Vi \\ SCHI Lab \\ University of Sussex, UK \\ C.Vi@sussex.ac.uk
}

\author{
Kasper Hornbæk \\ University of Copenhagen, \\ Denmark \\ kash@di.ku.dk
}

\author{
Sriram Subramanian \\ Interact Lab \\ University of Sussex, UK \\ Sriram@sussex.ac.uk
}

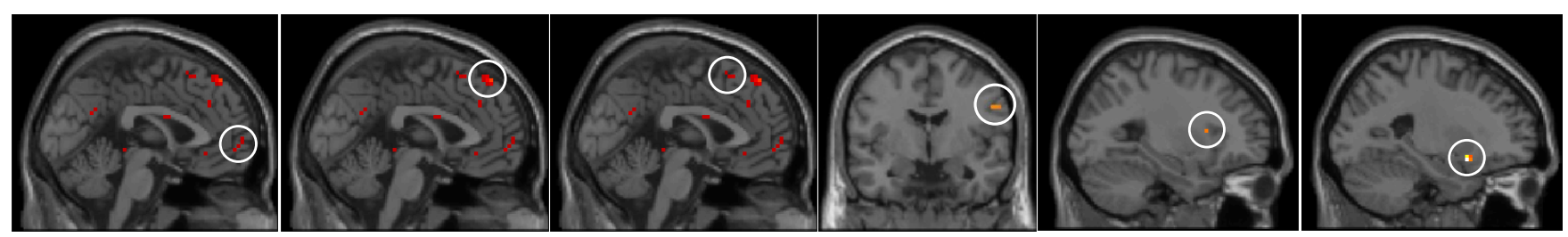

Figure 1. Some key regions of brain activity (in white circles) for assessment of perceived usability (left to right): left medial frontal gyrus, left superior frontal gyrus, right superior frontal gyrus, right precentral gyrus, left claustrum, and left putamen.

\section{ABSTRACT}

Usability has a distinct subjective component, yet surprisingly little is known about its neural basis and relation to the neuroanatomy of aesthetics. To begin closing this gap, we conducted two functional magnetic resonance imaging studies in which participants were shown static webpages (in the first study) and videos of interaction with webpages (in the second study). The webpages were controlled so as to exhibit high and low levels of perceived usability and perceived aesthetics. Our results show unique links between perceived usability and brain areas involved in functions such as emotional processing (left fusiform gyrus, superior frontal gyrus), anticipation of physical interaction (precentral gyrus), task intention (anterior cingulate cortex), and linguistic processing (medial and bilateral superior frontal gyri). We use these findings to discuss the brain correlates of perceived usability and the use of fMRI for usability evaluation and for generating new user experiences.

\section{Author Keywords}

User Experience, Aesthetics, Usability, fMRI

\section{ACM Classification Keywords}

H.5.2 [Information interfaces and presentation]: User Interfaces - Graphical user interfaces;

\section{INTRODUCTION}

Usability is a key concept in human-computer interaction (HCI) that concerns the ease, effectiveness, and satisfaction with which users achieve their goals when interacting with computers. Usability can be measured both objectively (e.g.,

Permission to make digital or hard copies of all or part of this work for personal or classroom use is granted without fee provided that copies are not made or distributed for profit or commercial advantage and that copies bear this notice and the full citation on the first page. Copyrights for components of this work owned by others than ACM must be honored. Abstracting with credit is permitted. To copy otherwise, or republish, to post on servers or to redistribute to lists, requires prior specific permission and/or a fee. Request permissions from permissions@acm.org. UIST 2017, October 22-25, 2017, Québec City, QC, Canada. (c) 2017 ACM. ISBN 978-1-4503-4981-9/17/10...\$15.00.

DOI: https://doi.org/10.1145/3126594.3126657 task completion time) and subjectively (e.g., asking users about their satisfaction) [43]. Such measures are important for evaluating computer systems and driving HCI research. For instance, improved usability increases the return on investment for information technology [53] and poor usability wastes billions of dollars every year [11].

The construct of usability, however, raises many open questions. For instance, the ISO 9241 standard names three aspects of usability, but others include different dimensions [40, 68, 79]. Thus, usability is not a clear construct. Moreover, while the relationship between usability and aesthetic appeal has been much researched (e.g., [7, 57, 58, 82]), one key question that remains is whether what is beautiful is also perceived as usable [57, 82], that is, whether and how immediate impressions of aesthetics influence subsequent performance/ assessment of usability.

We present an exploratory study of the neuroanatomical correlates of usability using functional magnetic resonance imaging (fMRI). The key motivation is to fill the gap in research on the neural basis of usability, and its similarities and differences to aesthetics. The study is exploratory and we hold no strong a priori hypotheses because (a) no prior work has used fMRI to investigate the neural basis of usability and (b) the literature on usability rarely hypothesize about relations or overlap among constructs.

The goal of this work is to link different components of usability to particular brain areas (and their underlying cognitive functions). We do so by comparing differences in brain activation during the judgment of aesthetics and usability, so as to identify the overlapping and uniquely activated brain areas and discuss associated cognitive functions. This allows us to make three contributions:

- We identify brain areas specific to usability and areas shared with aesthetics;

- We analyze usability theory based on an interpretation of the function of those brain areas; 
- We outline uses of fMRI for usability evaluation and for generating new user experiences.

\section{RELATED WORK}

Here, we will first discuss the construct of perceived usability and then review the principles of fMRI. Then we will highlight the lack of earlier fMRI studies on usability and describe the benefits of this line of work.

\section{Perceived Usability}

Usability as a construct has been widely accepted and disseminated in the HCI field. According to ISO [1], usability concerns the effectiveness, efficiency, and satisfaction with which users can achieve their goals when interacting with computers. Recently, there has been a surge of interest in user experience, bringing a focus on temporality and concepts such as the affective and the hedonic [38]. In addition, ISO [1] defines user experience as "a person's perceptions and responses that result from the use or anticipated use of a product, system or service". Following this definition, user experience includes many aspects of users' perceptions and experiences during and after use, including emotions, beliefs, and physical and psychological responses. Because of this, user experience has gained immense interest in HCI, as it highlights the non-utilitarian aspects of human-technology interactions, and focuses on user affect, sensation, and meaning as well as the value of such interactions in everyday life [55]. Although there is some controversy regarding the extent to which usability and user experience differ [8], we will refer to the construct of usability for the rest of the paper.

Despite the success and wide use of the usability construct, a variety of issues with its use persists (e.g., [56, 57]). First, usability is often considered to have different components or dimensions. The ISO 9241 standard, for instance, separates components of effectiveness, efficiency, and satisfaction; other definitions include different dimensions [40, 68, 79]. However, these dimensions correlate differently and sometimes only weakly [27]. And for some dimensions, many measures show unclear correlations [43].

Second, usability can be measured both objectively and subjectively. Examples of objective methods include usability testing and psychophysiological measurements (e.g., using functional near infrared spectroscopy (fNIRS) in usability testing $[41,42,60]$ and using electroencephalography (EEG) to evaluate visualization effectiveness [6] or to compare two user interfaces for managing personal photos and storytelling [85]). Examples of subjective methods include AttrakDiff2 [37] and the User Experience Questionnaire [54]. All are based on self-reports, collected verbally or non-verbally, and in-situ or post tasks. The relationship between subjective and objective measures is mixed, but some findings suggest much lower correlations than would be expected [27]. Even self-reports on usability may differ. Lindgaard and Dudek [57] found differences between ratings and interviews, suggesting that "rating scales and interview statements may tap different interface qualities."

Third, the specific relationship between usability and aesthetic appeal has been widely researched (e.g., [7, 57, 58, 82]). One key onus of this debate concerns whether "what is beautiful is usable" [57, 82], that is, whether and how immediate impressions of aesthetics influence subsequent performance and assessment of usability.

In sum, usability is widely used and beneficial to HCI. However, as a construct it remains evasive, with several open issues regarding exactly what usability is and how it compares to related concepts (e.g., aesthetics). Here, we limit the scope of user experience to perceived usability to help address in particular the first and third question above about the usability construct.

\section{Functional magnetic resonance imaging (fMRI)}

fMRI is a functional neuroimaging procedure that uses MRI technology to measure brain activity by detecting associated changes in blood flow [44]. The most common approach to fMRI uses the Blood Oxygenation Level Dependent (BOLD) contrast [44]. BOLD fMRI allows us to measure the ratio of oxy- $(\mathrm{oHb})$ to deoxy-haemoglobin $(\mathrm{dHb})$ in the blood. This does not directly measure neuronal activity. However, it does measure the metabolic demands (or oxygen consumption) of active neurons. Images constructed from fMRI during performance of different tasks reflect the parts of the brain that are active, and may reveal the brain structures that are activated together. This technique has higher spatial resolution and more accurate activation localization compared to EEG and fNIRS.

The human cerebral cortex is mapped by divisions into different functional areas known as Brodmann's areas. A Brodmann Area (BA) is a region of the cerebral cortex in the human brain, defined by its histological structure and organization of cells. Each BA can correlate with more than one cortical function and vice versa. For example, BA 1/ 2/ 3 are the primary somatosensory cortex which contains tactile representation of our body ([52]). In contrast, BA 10 occupies parts of superior frontal gyrus and the middle frontal gyrus. Therefore, reporting neural activations using fMRI should include information of both systems (cortical function area and BA number).

Functional MRI has been used in HCI to help understand cognitive functions during interactive tasks. Pine et al. [73], for example, analyzed the brain areas activated during the performance of a navigation task. Baumgartner et al. [9] studied brain activations associated with the experience of presence during a video of a virtual experience. A guide on conducting fMRIs studies can be found in [23].

Recent neuroimaging studies using fMRI have shown that user experience parameters might be reflected in brain activity measurements. In these studies, activated brain regions were investigated and associated with certain parameters. However, a common problem with interpreting 
$\mathrm{fMRI}$ is reverse inference, in which certain brain regions are alleged to indicate the activation of previously-labeled cognitive processes [23]. This problem arises because a particular brain region is seldom associated with only a single cognitive process [75]. Nonetheless, reverse inference is still useful in the discovery of interesting new facts about the mechanisms underlying a cognitive task. In fact, philosophers have previously argued that reverse inference (or 'abductive inference' in [71]), is an essential tool for scientific discovery $[75,76]$. We mention this because the later has to carefully guard against mistakes in inferences about brain areas related to perceived usability.

\section{Functional MRI studies of Usability and Aesthetics}

Due to its advantage of opening the "black box" underlying user experience, fMRI has been employed in several studies in HCI, especially for exploring virtual reality (VR). For example, Sjölie et al. [80] used fMRI to investigate the influence of two VR parameters, 3D-motion and interactivity for a mental rotation task. They found the most significant activations during the mental rotation task to be in the superior parietal lobe and occipital lobe. Clemente et al. [21] employed $\mathrm{fMRI}$ to investigate the sense of presence during a VR-free navigation task and found that frontal, parietal, and occipital regions were activated during the free virtual navigation. Anderson et al. [5] used fMRI to study users' habituation to security warnings. Their findings showed that the regions involved attentional processing (left and right superior parietal cortex) had higher activation for polymorphic than for static warnings, while the opposite was true in regions related to memory retrieval processes (bilateral medial prefrontal cortex and the left retrosplenial cortex). However, we are not aware of any study that has investigated the construct of usability or perceived usability using fMRI. Because of this, any first step of identifying the neural correlates of usability is exploratory, with no strong a priori hypothesis, rather than theory driven. This is similar to how fMRI studies on aesthetics started (e.g., [50]).

In contrast to usability, aesthetics has been investigated widely using fMRI to study the neural basis of the perception and experience of aesthetics. It has been shown that the pleasure elicited in people when they look at beautiful object is linked to general reward circuitry [51]. For example, when subjects passively viewed different types of faces, a significant effect was seen in the nucleus accumbens (NAc) $[2,50]$, an area close to the prefrontal cortex, and this effect was particularly seen in response to the more attractive female faces. In addition, activation of the caudate part of the striatum, which has an established role in processing reward related information [25], has been found to be correlated with the attractiveness of faces that held eye-contact with the subjects [47], and also with positive words [35]. This area, along with the cingulate gyrus and the middle frontal gyrus have been reported to be activated when judging the aesthetics of paintings [49, 83], pictures of soft drinks [69], and different types of faces [2]. A more detailed review can be found in [51] and [20].
In addition, as there might be a high correlation between the construct of usability and aesthetics, a topic that is currently being debated in HCI, an fMRI study will be helpful in answering this question. This can be done by comparing and contrasting the brain areas (and their cognitive functions) activated when looking at the perceived usability and aesthetics of the same type of visual stimuli.

\section{EXPERIMENT 1: BRAIN RESPONSES TO WEBPAGES}

The goal of Experiment 1 is to investigate whether differences in stimuli with respect to perceived aesthetics and perceived usability could be detected through fMRI scanning. We also wanted to investigate whether brain areas associated with perceived usability and with perceived aesthetics overlap. We looked at perceived usability and perceived aesthetics because (a) those are important to the discussion of the construct of usability, were prominent in reviews of usability measures (e.g., [8]), and were used in earlier studies of webpages (e.g., [59]) and (b) because they are feasible to study in an fMRI scanner, which has the benefit of evaluating users' perceptions (of which perceived usability is one element).

\section{Design}

Independent Variables and Design

The study looked at two factors, perceived usability and perceived aesthetics, with unique stimuli for each. The factors were manipulated through websites that scored high, medium, or low on these measures in a pre-study. Thus, the independent variables are perceived usability and perceived aesthetics, each with three levels (low, medium, and high).

\section{Selection of stimuli}

We collected 400 webpages as candidates for the stimuli to be used for the main study. The aim was to find web pages that "appear particularly usable, beautiful, stimulating or useful, or conversely, particularly unusable, ugly, dull, or irrelevant." All pages were in English and were captured as screenshots of $1920 \times 1080$ resolution. This procedure, including the rating described below, was similar to other studies on the visual perception of webpages [59, 67, 82].

On the crowdsourcing intermediary site CrowdFlower [22], 492 persons rated screenshots of webpages. Each rated five to fifteen pages on a variety of measures, including:

- $\quad$ Perceived usability, measured by a single question "How do you rate the usability of this web page?" (on a sevenpoint scale from "unusable" to "usable" [82]).

- Perceived aesthetics, measured by a single question, "How do you rate the beauty of the webpage?" (on a seven-point scale from "ugly" to "beautiful" as in [36]).

Participants also rated the webpages on their hedonic and pragmatic qualities (with eight questions from a shortened version of AttrakDiff2 [37]), goodness (as measured by a seven-point differential from "bad" to "good", as in [59]), and perceived aesthetics (measured by six questions from [81]. The questions covered so-called classical aesthetics, 
e.g., "clear", and expressive aesthetics, e.g., "sophisticated"). We did not use the last question (expressive aesthetics) except to check our selection of stimuli, as this would have required a further experimental construct. Participants could not interact with the webpages.

To select the stimuli to be used for the fMRI study, we ranked the webpages on the dimensions of perceived usability and perceived aesthetics separately to form three groups of the top $20 \%$ (80 webpages), middle $20 \%$, and bottom $20 \%$ of each dimension. To ensure the least interaction between measures, we selected 25 webpages from each group that had the greatest difference in rank between perceived usability and perceived aesthetics, for a total of 150 unique webpages (Table 1). Here the correlations between perceived beauty and usability were similar, as found in previous studies (e.g., Tractinsky et al. [81], Tuch et al. [82]). Thus, the two attributes seem to be consistently related. In this design we use different pages for ratings of beauty and usability so that recognizing a stimuli would not generate spurious brain activations.

\begin{tabular}{|c|c|c|c|c|c|c|}
\hline \multirow{2}{*}{} & \multicolumn{3}{|c|}{ Beauty (N=75) } & \multicolumn{3}{c|}{ Usability (N= 75) } \\
\cline { 2 - 7 } & Low & Med & High & Low & Med & High \\
\hline Perceived & 4.63 & 4.99 & 5.37 & 3.89 & 5.24 & 6.08 \\
Usability & $(.51)$ & $(.40)$ & $(.36)$ & $(.52)$ & $(.14)$ & $(.23)$ \\
\hline Perceived & 3.20 & 4.89 & 5.98 & 4.37 & 4.04 & 5.07 \\
Aesthetics & $(.47)$ & $(.12)$ & $(.17)$ & $(.52)$ & $(.55)$ & $(.51)$ \\
\hline
\end{tabular}

Table 1. Average ratings (with standard deviation) of webpages selected for the fMRI study.

\section{Functional MRI scanning method}

\section{Participants}

Eight right-handed participants (4 males; mean age $27.4 \pm$ 6.0) participated in the experiment. The participants were screened using a clinical questionnaire to ensure that none had a current or prior history of head injury, learning disability, or psychiatric illness. All participants were reported to be free of psychotropic medication and had normal or corrected-to-normal vision. Each participant gave written informed consent after the explanation of the experimental protocol, as approved by the local Ethics committee. The participants were paid $\$ 15$.

\section{Procedure}

Perceived usability and perceived aesthetics were separated in the experimental design and treated as two separate investigated factors (see Figure 3). For each factor, webpages of three levels were shown randomly to participants. This followed previous studies (e.g., [46]) and was also done to reduce the boredom of participants if webpages of the same category are shown continuously.

As a consequence, participants viewed a total of 150 webpages representing two factors (perceived aesthetics and perceived usability). Each factor had three levels (Low, Neutral, and High), and each level had a group of 25 webpages. Webpages for all levels of a factor (75 in total) were presented in a random order between levels. The order of presentation of factors (one followed by the other) was counter-balanced between participants. The participants were instructed before viewing the webpages for each factor that they would be rating either aesthetics or usability. The instruction was provided on a screen with only the word BEAUTY or USABILITY at the screen's center for $2 \mathrm{~s}$. All webpages were converted into 1024 × 768 resolution to match the resolution of the fMRI display.

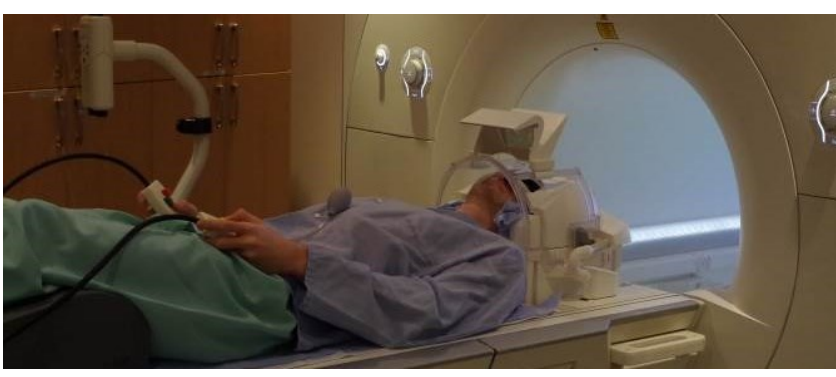

Figure 2. Setup of a participant just before entering the fMRI machine. The participant had two keypads with two keys each, and a head mounted mirror to see the screen at the back.

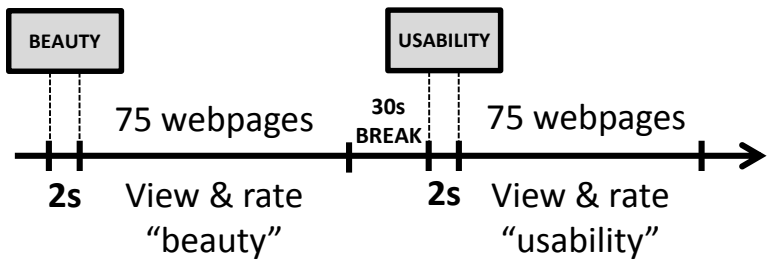

Figure 3. Procedure for one experiment, where the beauty (i.e., perceived aesthetics) was viewed and rated first.

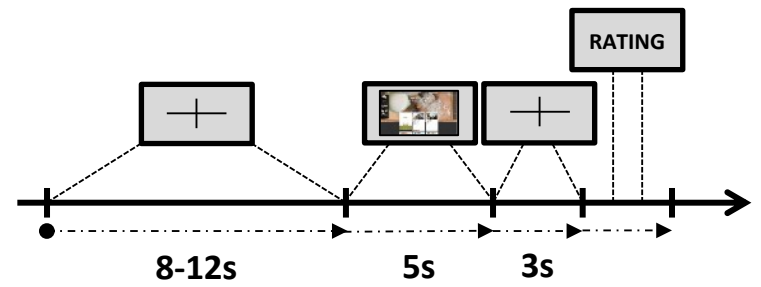

Figure 4. Procedure for one trial

Each trial started with a fixation cross displayed at the middle of a blank screen for a random duration (Inter-Stimulation Interval - ISI) between 8 and 12s. This randomized ISI was designed to reconstruct the BOLD response with better precision. Next, a webpage was shown for $5 \mathrm{~s}$, which gave the participant enough time to observe and form an opinion. A blank screen with a cross at the middle was then displayed for $3 \mathrm{~s}$, after which the participants were asked one of the two questions corresponding to the factor being tested:

or

$$
\begin{gathered}
\text { Rate the usability of the last shown picture } \\
\text { Low Neutral High }
\end{gathered}
$$

Rate the beauty of the last shown picture
Low Neutral High

The participants were given unlimited time to answer the question for each webpage. There was a break of 30s between the ratings of perceived aesthetics (beauty) and 
perceived usability (usability), see Figure 3. Figure 4 shows the timing of each trial. Participants were instructed to rate the perceived aesthetics of a webpage by its overall visual attractiveness and appeal, and to rate perceived usability of a webpage by its ease of use and navigation, and ease by which information could be obtained. The stimuli exposure time (5s) follows other works that have presented visual stimuli in fMRI [5, 30, 45]. Jacobs et al. [45] argued that longer presentation time of visual stimuli lead to activation patterns associated with a deeper processing of the stimuli. However, we had to balance the duration of the scanning ( 1 hour) and the number of stimuli to be presented.

After being in the MRI scanner, participants went through all the webpages in the stimuli set again and inputted their detailed ratings, using the same questionnaires as in the CrowdFlower pre-study. This was done because the participants who performed the fMRI scanning were different from those who completed the pre-study, and allowed us to check if answers were consistent between the two groups. The questionnaire was completed on a laptop, outside the MRI scanning room. Participants entered their answers on a 7-point Likert scale, as in Figure 5.

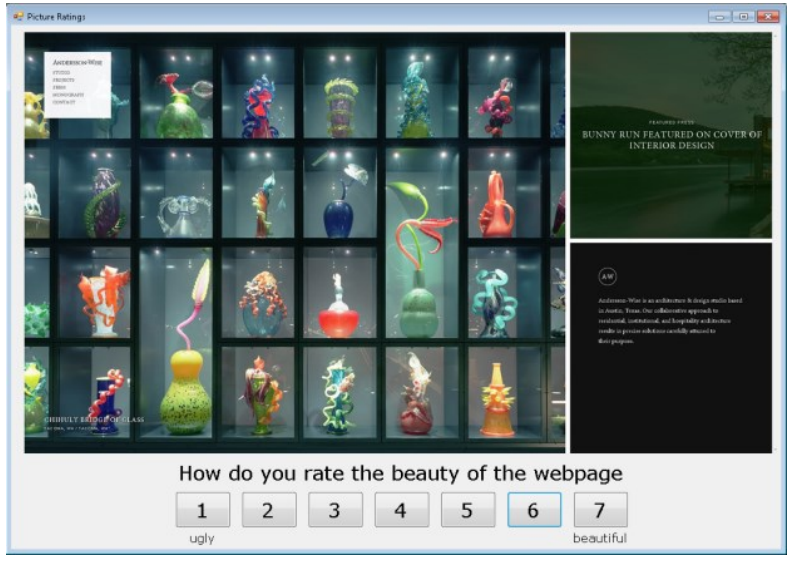

Figure 5. Post-scan webpage rating task

The experiment lasted about $1 \mathrm{~h} 15 \mathrm{~m}$ per participant, with $60 \mathrm{~m}$ scanning and $15 \mathrm{~m}$ for the post-scanning questionnaire.

\section{MRI Data Acquisition}

Experiments were run with the Psychophysics Toolbox [14]. Neuroimaging data was acquired with a 3T Siemens Magnetom Skyra MRI scanner. Subjects were placed in a 32channel RF head coil with a mirror mounted on the coil to view the webpages. Soft padding was placed on either side of the head to limit head movement during fMRI image acquisition. Webpages were presented on a screen mounted at the back of the scanner, which participants could observe through the attached head-mounted device. Ratings of webpages were collected using a 4-key fiber-optic Lumina response box (see Figure 2). Functional images were acquired with the following parameters for $\mathrm{T} 2 *$-weighted gradient echo sequence: $64 \times 64$ matrix, TR $=2,500 \mathrm{~ms}, \mathrm{TE}=$ $30 \mathrm{~ms}, \mathrm{FOV}=192 \mathrm{~mm}$, flip angle 90 . Thirty-six slices were acquired to cover the whole brain with an interleaved slice acquisition and $3 \times 3 \times 3 \mathrm{~mm}$ voxel resolution. In addition, a high resolution structural scan was acquired with T1weighted MP-RAGE sequence (256 x 256 matrix, 192 slices in sagittal plane and a $0.9 \times 0.9 \times 0.9 \mathrm{~mm}$ voxel).

\section{Analysis Method}

Statistical Parametric Mapping software (SPM8; Welcome Trust Centre for Neuroimaging) was used for image data processing and analysis. The first seven scans were excluded from the analysis to eliminate the decay of the fMRI signal associated with the moment when magnetization reaches equilibrium. Functional data from each run were aligned to the run nearest in time to the acquisition of the structural scan. Then, the mean image produced during the process of realignment, and the realigned images were co-registered to the high-resolution T1 anatomical image. All images were spatially normalized to standard MNI space. They were then spatially smoothed using 10-mm FWHM Gaussian kernel to facilitate group analysis, and a high-pass filter of $1 / 128 \mathrm{~Hz}$ was used to eliminate low-frequency components. The functional imaging data were modeled using a boxcar function with head motion parameters as unrelated regressors. Parameter estimates for each condition (three types of stimuli) were calculated from a general linear model (GLM) based on the hemodynamic response function with overall grand mean scaling. Whole-brain statistical parametric mapping analyses were performed. The t-contrast images were generated for comparison at each voxel. Statistical tests were first assessed in individual subjects, and then random effect analyses were conducted based on statistical parameter maps from each individual subject, to allow population inference. A one-sample t-test was applied to determine group-level activation for intelligibility effect. This process was similar to previous works [26, 29].

\section{RESULTS OF EXPERIMENT 1}

\section{Behavioral results}

On average, it took participants $0.93 \mathrm{~s}(S D=0.49 \mathrm{~s})$ to rate a webpage in the scanner. A t-test shows no significant difference in response time between perceived aesthetics $(M$ $=0.90 \mathrm{~s}, S D=0.71 \mathrm{~s})$ and usability $(M=0.96 \mathrm{~s}, S D=0.79 \mathrm{~s}), p$ $=0.22$. An ANOVA with Bonferroni correction shows no significant difference in response time within the perceived aesthetics groups $(p=0.67)$. However, participants spent less time rating high usability webpages compared to low usability ones $(p<0.005)$.

We compared the ratings obtained from CrowdFlower (called cloud ratings), from participants while inside the scanner (called inscan ratings), and from participants in the post-scan task (called postscan-ratings). A Pearson's correlation test between cloud-, inscan-, and postscan ratings shows that participants were fairly consistent in rating webpages during and after the scans $(r=0.74)$. However, it also shows that judgment regarding the perceived usability of a webpage is fairly personal, with cloud- and inscan ratings having $r=$ 0.52 . Figure 6 illustrates these different types of responding time. 


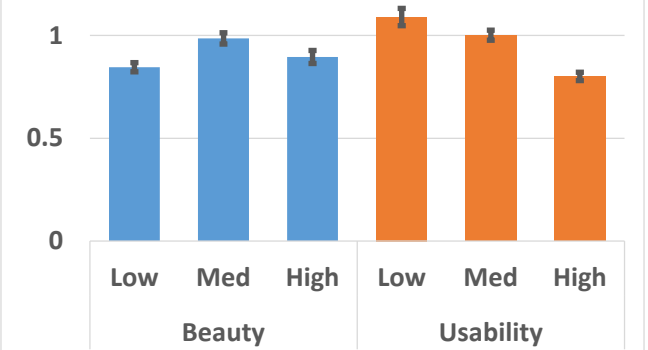

Figure 6. Inscan responding time with standard error bars.

\section{Testing of Brain Activity}

To analyze the fMRI data, we contrasted conditions of interest corresponding to each level of perceived aesthetics and usability by assigning values of 1 and -1 to the regressors of interest, and 0 to all other regressors. The brain activity occurring with when there were no stimuli presented was used as baseline condition and was assigned a value of 0 in all contrasts. Following the experimental design, we analyzed perceived aesthetics and usability separately.

Specifically, we first did pair-wise comparisons between baseline and stimuli for aesthetics and usability. We then did pair-wise contrasts of brain regions that were active during the different levels of usability and aesthetics (e.g., low vs. high). That helped to identify regions involved in different levels of usability and aesthetics.

\section{Brain Correlates of Perceived Aesthetics}

We observed a large number of activation areas across the brain during the judgment of perceived aesthetics. Table 2 shows the details of these areas for the contrasts of perceived aesthetics vs. baseline. We found several regions in frontal lobe, temporal lobe, limbic lobe, and basal ganglia that were activated in the contrast of perceived aesthetics vs. baseline $(p<0.001)$. Table 2 also shows the details of activation areas for the comparisons of different levels of perceived aesthetics (low/medium/high). We found activations in the frontal lobe, parietal lobe, and the limbic lobe for both the high vs. low and high vs. medium comparisons of perceived aesthetics.

\section{Discussion of Perceived Aesthetics}

The brain areas found to be specific to perceived aesthetics are in line with those found for non-webpage stimuli. For instance, we observed strong activation in the left middle frontal gyrus, suggesting that people rate the perceived aesthetics of webpages similarly to pictures and architecture stimuli $[46,84]$. This may be because most webpages include commodity design elements such as columns, frames and headings. These commodity design elements add architectural features to the static webpage, thus capturing users' attention. In addition, the activations seen in the bilateral inferior frontal gyrus, claustrum, and insula are similar to a previous study demonstrating baby schema [30], suggesting that the participants may perceive the features of webpages as cute or motivating a sense of caretaking in the subjects. Activation of the sub-gyral, an area associated with the hippocampus [18], suggests that the perceived aesthetics of webpages are also judged based on visual aesthetic perception. Thus, our study found activation areas related to perceived aesthetics that were also reported in previous studies involving aesthetic stimuli.

\begin{tabular}{|c|c|c|c|c|c|c|c|}
\hline Structure & & BA & k & $\mathbf{x}$ & y & $\mathbf{z}$ & $\mathbf{p}$ \\
\hline \multicolumn{8}{|c|}{ Aesthetics - baseline } \\
\hline \multicolumn{8}{|l|}{ Frontal lobe } \\
\hline Middle Frontal Gyrus & L & 10 & 168 & -40 & 42 & 14 & $1.3 \mathrm{E}-5$ \\
\hline Inferior Frontal Gyrus & $\mathrm{R}$ & $45 / 47$ & 68 & 49 & 20 & 11 & 0.0001 \\
\hline Medial Frontal Gyrus & $\mathrm{R}$ & 9 & 27 & 5 & 44 & 18 & 0.0002 \\
\hline Medial Frontal Gyrus & L & 10 & 27 & -1 & 47 & 12 & 0.0004 \\
\hline \multicolumn{8}{|l|}{ Temporal lobe } \\
\hline Superior Temporal Gyrus & $\mathrm{L}$ & 22 & 41 & -45 & -5 & -4 & $1.6 \mathrm{E}-5$ \\
\hline \multicolumn{8}{|l|}{ Limbic lobe } \\
\hline Anterior Cingulate & $\mathrm{R}$ & 24 & 260 & 1.8 & 86 & 5.44 & $9.9 \mathrm{E}-6$ \\
\hline Insula & $\mathrm{R}$ & 13 & 103 & 43 & -6 & 3 & $3.5 \mathrm{E}-5$ \\
\hline Insula & L & 13 & 151 & -43 & -1 & 3 & $4.5 \mathrm{E}-5$ \\
\hline Sub-Gyral (Hippocampus) & $\mathrm{R}$ & & 28 & 35 & -4 & 4 & $3.9 \mathrm{E}-5$ \\
\hline Cingulate Gyrus & $\mathrm{L}$ & 23 & 25 & 1.4 & -2 & 24 & 0.0002 \\
\hline Cingulate Gyrus & $\mathrm{R}$ & 31 & 25 & 1.2 & -3 & 32 & $6.9 \mathrm{E}-5$ \\
\hline \multicolumn{8}{|l|}{ Basal ganglia } \\
\hline $\begin{array}{l}\text { Lentiform Nucleus } \\
\text { Putamen }\end{array}$ & L & & 151 & -29 & -1 & -2 & 0.0005 \\
\hline Claustrum & $\mathrm{L}$ & & 110 & -26 & -13 & 22 & $7.8 \mathrm{E}-6$ \\
\hline Caudate (Caudate Tail) & $\bar{R}$ & & 28 & 29 & -3 & 8 & 0.0007 \\
\hline \multicolumn{8}{|c|}{ Aesthetics (High - Low) } \\
\hline \multicolumn{8}{|l|}{ Frontal lobe } \\
\hline Middle Frontal Gyrus & L & 46 & 12 & -46 & 19 & 20 & $2.5 \mathrm{E}-5$ \\
\hline \multicolumn{8}{|l|}{ Parietal lobe } \\
\hline Angular Gyrus & $\mathrm{L}$ & 39 & 6 & -29 & -62 & 31 & $1.8 \mathrm{E}-5$ \\
\hline Precuneus & $\mathrm{L}$ & 19 & 6 & -32 & -68 & 39 & 0.0007 \\
\hline \multicolumn{8}{|l|}{ Limbic lobe } \\
\hline Cingulate Gyrus & $\mathrm{R}$ & 24 & 5 & 4.12 & 5.23 & 38.17 & 4.4E-5 \\
\hline \multicolumn{8}{|c|}{ Aesthetics (High - Med) } \\
\hline \multicolumn{8}{|l|}{ Frontal lobe } \\
\hline Medial Frontal Gyrus & $\mathrm{R}$ & 6 & 50 & 1 & 1 & 57 & 0.0002 \\
\hline Medial Frontal Gyrus & L & 6 & 8 & -7 & 0 & 59 & 0.0002 \\
\hline Paracentral Lobule & $\mathrm{R}$ & 31 & 10 & 4 & -13 & 47 & 0.0003 \\
\hline Paracentral Lobule & L & 31 & 7 & -2 & -21 & 46 & 1.3E-5 \\
\hline \multicolumn{8}{|l|}{ Parietal lobe } \\
\hline Inferior Parietal Lobule & L & 40 & 7 & -57 & -27 & 26 & 0.0002 \\
\hline Inferior Parietal Lobule & $\mathrm{R}$ & 40 & 7 & 57 & -29 & 36 & 4.0E-5 \\
\hline \multicolumn{8}{|l|}{ Limbic lobe } \\
\hline Cingulate Gyrus & $\mathrm{R}$ & 24 & 11 & 1.31 & 4.98 & 40.81 & 0.0002 \\
\hline Cingulate Gyrus & L & 24 & 10 & -7.1 & -14.8 & B 41.49 & 0.0002 \\
\hline
\end{tabular}

Table 2. Regions activated in the parametric analyses of inscan ratings for perceived aesthetics. Here, $L / R$ refers to the Left/ Right cerebrum of the brain; BA refers to the Brodmann area number; $k$ is the cluster size of the activation, measured in the number of voxels; $x, y, z$ are coordinates in Talairach space; and $p$ is the probability of the comparison.

Brain Correlates of Perceived Usability

Similar to the findings for perceived aesthetics, the comparison of Perceived Usability vs. baseline revealed activations across the brain in the frontal, temporal, and limbic lobes $(p<0.001)$. The activated areas are shown in Figure 1 and Table 3, and are discussed further in the next paragraph. Specifically, we found that in some areas in the frontal, parietal, and limbic lobes, and in the basal ganglia, responded to the differences in perceiving different levels (high, medium, and low) of usability. Here, only the high vs. low and medium vs. low comparisons of perceived usability yielded activations $(p<0.001)$, while no significant activation was found for high vs. medium. 


\section{Discussion of Perceived Usability}

Perceived usability was found to be associated with increased activation of several premotor areas, including the superior frontal gyrus (BA6) and the precentral gyrus (BA6). The latter showed increased activation with increased usability. One interpretation of BA6 is that it is associated with planning complex movements, as it is the site of the premotor cortex and supplementary motor cortex [19]. Similarly, the right superior frontal gyrus (BA10) is involved in planning to use something [19]. In addition, the right superior frontal gyrus (BA10) was found to be activated in a previous study of reward in an N-back task (where subjects watch a sequence of stimuli and need to recall whether the current stimulus was the same as the previous $\mathrm{N}^{\text {th }}$-stimulus) [74]. The region was also found to be activated in a self-aware state [31]. This suggests that the process of planning to use a webpage and self-reflection on how to use the webpage viewed were stimulated. The activation of these areas was specific to usability.

\begin{tabular}{|c|c|c|c|c|c|c|c|}
\hline Structure & & BA & $\mathbf{k}$ & $\mathbf{x}$ & y & $\mathbf{z}$ & $\mathbf{p}$ \\
\hline \multicolumn{8}{|c|}{ Usability - baseline } \\
\hline \multicolumn{8}{|l|}{ Frontal lobe } \\
\hline Middle Frontal Gyrus & $\mathrm{L}$ & 9 & 30 & -32 & 26 & 29 & $7.3 \mathrm{E}-5$ \\
\hline Medial Frontal Gyrus & $\mathrm{L}$ & $9 / 10$ & 26 & -4 & 56 & 10 & 0.0005 \\
\hline Medial Frontal Gyrus & $\mathrm{R}$ & 8 & 16 & 1 & 28 & 38 & $2.1 \mathrm{E}-5$ \\
\hline Superior Frontal Gyrus & $\mathrm{L}$ & 8 & 24 & -2 & 35 & 54 & $1.8 \mathrm{E}-5$ \\
\hline Superior Frontal Gyrus & $\mathrm{R}$ & 6 & 12 & 4 & 15 & 55 & 0.0001 \\
\hline \multicolumn{8}{|l|}{ Temporal lobe } \\
\hline Middle Temporal Gyrus & $\mathrm{R}$ & $19 / 37$ & 22 & 35 & -57 & 14 & 0.0001 \\
\hline \multicolumn{8}{|l|}{ Limbic lobe } \\
\hline Cingulate Gyrus (31) & $\mathrm{L}$ & 31 & 62 & -21 & -45 & 30 & $4.6 \mathrm{E}-6$ \\
\hline Posterior Cingulate (31) & $\mathrm{R}$ & 31 & 20 & 1.29 & -58 & 24 & 0.0001 \\
\hline \multicolumn{8}{|c|}{ Usability (High - Low) } \\
\hline \multicolumn{8}{|l|}{ Frontal lobe } \\
\hline Superior Frontal Gyrus & $\mathrm{R}$ & 10 & 17 & 27 & 49 & 26 & 0.0001 \\
\hline Fusiform Gyrus & $\mathrm{L}$ & 37 & 11 & -46 & -60 & -12 & $2.3 \mathrm{E}-5$ \\
\hline Cingulate Gyrus & $\mathrm{R}$ & 24 & 11 & 4 & 2 & 41 & $3.8 \mathrm{E}-5$ \\
\hline Precentral Gyrus & $\mathrm{R}$ & 6 & 5 & 49 & -8 & 32 & 0.0004 \\
\hline \multicolumn{8}{|l|}{ Limbic lobe } \\
\hline Cingulate Gyrus & $\mathrm{L}$ & 32 & 7 & -1 & 23 & 34 & 0.0007 \\
\hline Cingulate Gyrus & $\mathrm{R}$ & 32 & 7 & 7 & 23 & 34 & $7.4 \mathrm{E}-5$ \\
\hline Anterior Cingulate & $\mathrm{R}$ & 32 & 7 & 7 & 26 & 27 & 0.0001 \\
\hline \multicolumn{8}{|l|}{ Parietal lobe } \\
\hline Inferior Parietal Lobule & $\mathrm{L}$ & 40 & 9 & -49 & -32 & 47 & 0.0001 \\
\hline Postcentral Gyrus & $\mathrm{L}$ & 2 & 9 & -52 & -23 & 45 & 0.0006 \\
\hline \multicolumn{8}{|l|}{ Basal ganglia } \\
\hline Lentiform Nucleus / & $\mathrm{L}$ & & 8 & -24 & 10 & -8 & $4.6 \mathrm{E}-5$ \\
\hline Claustrum & $\mathrm{L}$ & & 8 & -23 & 22 & 10 & 0.0001 \\
\hline \multicolumn{8}{|c|}{ Usability (Med - Low) } \\
\hline \multicolumn{8}{|l|}{ Parietal lobe } \\
\hline Inferior Parietal Lobule & $\mathrm{L}$ & 40 & 5 & -41 & -45 & 38 & $1.4 \mathrm{E}-5$ \\
\hline
\end{tabular}

Table 3. Regions activated in the parametric analysis of inscan ratings for perceived usability.

Usability was also related to activation of the medial and bilateral superior frontal gyri (BA8). Activation of these areas was specific to perceived usability, and they are often related to linguistic processing and reading [66]. In some studies, they have also been implicated in higher-order expectancy and utility [17]. This suggests that even if a webpage is only viewed for $5 \mathrm{~s}$, users look at the quality of the headings in the webpage when perceiving usability.
Increases in usability were also found to be associated with activation in areas of the somatosensory cortex associated with touch (postcentral gyrus, [77]). This has been seen in the process of observing touch actions [12], which is a kind of "mirror effect" of touch. This effect was observed in our study, suggesting that nowadays webpages are often viewed on a touch-based device, which might lead participants to assess the usability of a webpage by asking themselves: "What happens if I go there (by touching it)?"

Finally, the left middle frontal gyrus (BA9) was activated when assessing usability. This region is related to emotion [23] and was specific to perceived usability. This area has been shown to be related to the implementation of reappraisal (the emotional regulation strategy that involves changing the trajectory of an emotional response) in daily life [32]. This suggests that the affective influence of a webpage is a key factor in perceiving usability.

\section{Discussion of Experiment 1}

The results from this experiment show an overlap in brain activation when perceiving aesthetics and usability, in the left medial frontal gyrus (BA 10) and the inferior parietal lobule (BA 40). The left medial frontal gyrus was likely activated in response to commodity design elements when subjects were asked to judge the usability of a static webpage. This effect has also been seen in the parametrical effects of aesthetics and familiarity ratings [13] and aesthetic judgment of pictures [46]. Also, the activation of the inferior parietal lobule has been observed in response to infant faces versus crosshair baby schema levels. The overlap in these regions between aesthetics and usability may be interpreted in several ways. First, it may be that the assessment of some components of perceived usability is replaced by the (presumably simpler) assessment of perceived aesthetics. Second, perceived aesthetics may form part of perceived usability, causing the activation areas to overlap.

The results described above show brain activation areas similar to those found in previous studies. This suggests that the participants perceived the webpages in a similar way to non-webpage stimuli. However, activation of these areas has been observed in a combination of several studies of different types of stimuli (paintings, landscapes, faces, etc.). This suggests that the participants perceived the aesthetics of webpages as a combination of separate components, according to the different components of the webpage itself. Similarly, because of the overlap between perceived aesthetics and usability, it can be suggested that participants also perceived usability of webpages as a combination of the different components' aesthetics and usable values of the being-viewed webpages.

\section{EXPERIMENT 2: DYNAMIC STIMULI}

In Experiment 1, we found that viewing beautiful webpages triggers similar activation areas in the brain as have been found in previous studies. These same areas were also activated when participants viewed webpages with low usability. Many models of usability acknowledge that initial 
exposure to an interface differs from after interacting with it (e.g., [3, 48]). For example, measures of aesthetics and of usability are often strongly correlated at the time of initial exposure to an interface, but less correlated - if at all - after interaction with an interface. Thus, while the first study presented initial data on the associations between activities in brain networks and usability and aesthetic ratings, these relationships might differ in interactive systems.

To investigate this further, we added dynamics to the stimuli and performed the same experimental protocol. This was done to check whether people evaluate perceived aesthetics and usability in an interactive user interface the same as with static webpages (where there is no interactivity between the application and the user). Interactive here refers to interfaces that change appearance, as demonstrated by videos showing the user interface changing, either through a fictive user interacting with it (indicated through the mouse moving) or to show a notification. This approximates real interaction, and earlier work on user interfaces has used this approach ([70]). Furthermore, previous works in neuroscience suggests that at least some reactions to observing people use tools are similar to the reactions people have when using the tool themselves (e.g., [64, 78]).

\section{Design \& Method}

\section{Selection of Stimuli}

From Experiment 1's results, we saw that the BOLD signal response for the medium group was not significant different from the activation of the high group (usability) or low group (aesthetics) and with limited contrast between them. Consequently, in this experiment, we created videos of simple webpage interactions based on 100 webpages from the previous experiment (25 from each group: low aesthetics, high aesthetics, low usability, high usability). In addition, we added 10 additional videos to each group that clearly showed low/high aesthetics or usability. This was done to increase the activation-based contrast by having a larger number of samples for each group.

Each video contained a normal webpage interaction such as the mouse scrolling down, or a mouse click to change page (1-2 times). The mouse cursor and its trajectories were visible in the video. All animations were captured using Camtasia Studio Screen Recorder and saved in MP4 and $1080 p$ video format. They were later converted to $1280 \times 720$ size (720p) to fit the MRI projector resolution. On average, the videos had a length of $7.56 \mathrm{~s}( \pm 1.19 \mathrm{~s})$.

\section{fMRI Procedure}

We employed the same procedure for video stimuli as with the previous experiment on static webpages (Figure 3 and Figure 4). In short, there was a blank screen with a cross displayed at the middle for 8-12s, followed by the video. After that, the same blank screen appeared for $3 \mathrm{~s}$, and then a question popped up to ask the participants for a rating of the video. Similar to the previous experiment, participants were instructed to rate the perceived aesthetics of the webpage by its overall visual attractiveness and appeal, and rate perceived usability by its ease of use and navigation, and ease of obtaining information.

Eight participants (4 male), aged from 18 to 32, volunteered for this experiment. They were all right-handed (by selfreport) and did not participate in the previous experiment. Similar to the previous experiment, the participants completed a clinical questionnaire to ensure that none had a current or prior history of head injury, learning disability, or psychiatric illness. All participants were free of psychotropic medications and had normal or corrected-to-normal vision. They were paid $\$ 15$ for their time. None of the volunteers had participated in the previous experiment.

Also similar to Experiment 1, after being scanned in the MRI scanner, the participants went through all videos in the stimuli set and inputted their detailed ratings. This was done to see whether the answers were consistent between the experimental and pre-test groups. The questionnaire was completed on a laptop, outside the MRI scanning room. Participants entered their answers on a more detailed scale (7-point Likert scale). The total experiment time for each participant was about 1 hour 15 minutes, with 1 hour for scanning and 15 minutes for the post-scan questionnaire.

\section{Results}

\section{Behavioral}

On average, it took participants $1.07 \mathrm{~s}( \pm 1.23 \mathrm{~s})$ to respond to a rating question in the scanner. ANOVA with Bonferroni correction shows a difference in response time between the perceived aesthetics and usability groups $(\mathrm{p}<0.05)$. Participants spent a slightly longer time to judge the perceived usability of the stimuli (mean 0.996s \pm 0.226 ) compared to the perceived aesthetics (mean 1.155s \pm 0.226 ). A post-hoc test reveals no significant difference between high and low ratings within each group of perceived aesthetics and usability $(p>0.05)$.

\section{Brain activity for Perceived Usability and Aesthetics}

We used a similar method as in the first study to contrast the conditions of interest corresponding to each level of aesthetics and usability by assigning values of 1 and -1 to the regressors of interest, and 0 to all other regressors. We also analyzed aesthetics and usability separately.

Table 4 shows the activation areas for the contrasts of perceived aesthetics vs. baseline and perceived usability vs. baseline. We did not find significant activation areas $(\mathrm{k}>=4)$ when comparing different levels of perceived aesthetics and usability. We observed activations in the contrast of perceived aesthetics vs. baseline in the frontal lobe (bilateral medial frontal gyrus, BA6, and the precentral gyrus, BA6). For perceived usability vs. baseline, there was increased activity in the precentral gyrus (BA4). Interestingly, all activations related to aesthetics were located in BA6, which is the site of the premotor cortex and supplementary motor cortex. This area is related to early planning of movements and events, as discussed earlier. In contrast, the activations 
caused by usability vs. baseline were found in the precentral gyrus (BA4), which was not activated for perceived aesthetics (Figure 7). This is the site of the primary motor cortex, which controls all voluntary movements. This suggests that perceived usability is somewhat more related to action than perceived aesthetics.

\begin{tabular}{|c|c|c|c|c|c|c|c|}
\hline Structure & & BA & k & $\mathbf{x}$ & $\mathbf{y}$ & $\mathbf{z}$ & $\mathbf{p}$ \\
\hline \multicolumn{8}{|c|}{ Aesthetics - baseline } \\
\hline \multicolumn{8}{|l|}{ Frontal lobe } \\
\hline Medial Frontal Gyrus & $\mathrm{R}$ & 6 & 19 & 15 & -16 & 53 & 0.0003 \\
\hline Medial Frontal Gyrus & L & 6 & 4 & -13 & -13 & 55 & 0.0002 \\
\hline Precentral Gyrus & $\mathrm{R}$ & 6 & 5 & 43 & -12 & 35 & $6.2 \mathrm{E}-5$ \\
\hline \multicolumn{8}{|l|}{ Basal ganglia } \\
\hline Caudate Tail & $\mathrm{L}$ & & 5 & -21 & -37 & 12 & 0.0002 \\
\hline \multicolumn{8}{|c|}{ Usability - baseline } \\
\hline \multicolumn{8}{|l|}{ Frontal lobe } \\
\hline Precentral Gyrus & $\mathrm{R}$ & 4 & 14 & 23 & -22 & 58 & $1.4 \mathrm{E}-5$ \\
\hline
\end{tabular}

Table 4. Regions activated in the parametric analyses of inscan ratings for perceived aesthetics and usability when viewing animated webpages.

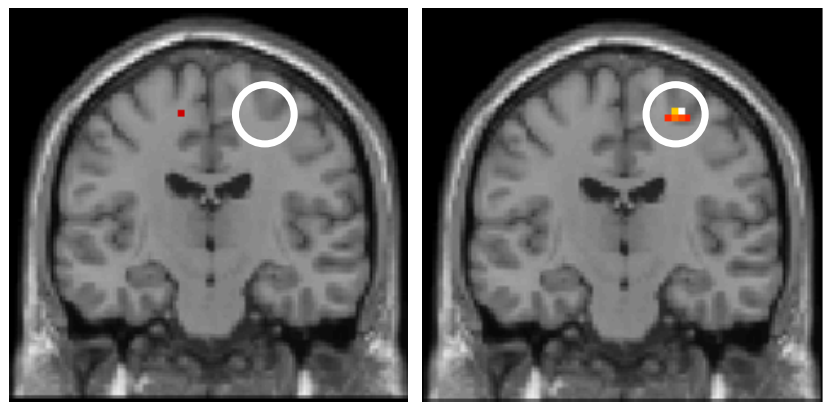

Figure 7. Activations in the precentral gyrus (BA4) for perceived aesthetics vs. baseline (left) and perceived usability vs. baseline (right).

\section{Discussion of Experiment 2}

The results show that for the contrast of perceived aesthetics vs. baseline, the bilateral medial frontal gyrus was activated. Its activation has been found during observation of cartoons and story comprehension involving animation stimuli [28]. Also, activations in the caudate tail have been shown to be associated with 'what' and 'where' the information was received and guides the rapid eye movement to visual objects [86]. This area related to eye movements is likely linked to the recorded interaction embedded in the animated webpages. This result suggests that participants assessed not only the perceived aesthetics of the webpage's but also the interaction.

\section{OVERALL DISCUSSION}

Our results show that there are distinct differences between the neuroanatomical areas related to perceived usability and those related to perceived aesthetics. We have shown this both as differences between a baseline condition and viewing of webpages, and as differences between seeing webpages that have been assessed as high, medium, or low on perceived usability and aesthetics. We have also shown that recorded interactivity added to webpages attenuates these differences and allows fewer distinct brain areas to be discerned. Below, we discuss these results.
The brain components of perceived usability

A key finding of this paper is that perceived usability has a brain basis that differs from that of perceived aesthetics. We find this remarkable because (a) exposure time to webpages was limited, (b) only static images were shown in Experiment 1, and (c) many studies find moderate correlations between assessments of perceived usability and perceived aesthetics (e.g., [82]). Still, there is something particular to usability that emphasizes its prominent status in human-computer interaction. We are particularly excited about four connections in brain areas specific to usability.

First, our results suggest that emotion is integral to perceived usability. We found activation in the fusiform gyrus, which is involved in emotional facial expression, and in the middle frontal gyrus (BA9), which shows activity during emotional suppression [4]. Another evidence is the activation seen in the superior frontal gyrus (BA10) and the anterior cingulate gyrus in the contrast of Usability vs. baseline. Both have been found to show activations during the self-regulation of emotion [10]. In contrast, many current conceptions of usability do not clearly specify a role for emotion in usability (e.g., ISO 9241); our findings suggest that emotion does indeed play a part.

Second, the findings of the first experiment suggest that the anticipation of physical interaction is a crucial part of perceived usability. During the judgment of usability of static webpages, the precentral gyrus (BA6) was activated, which may relate to the planning of movements related to the webpage. Interestingly, some studies relate this area to the sense of touch; others to the notion of affordance [24]. In the second experiment, during the judgment of videos of webpages, some activation was found in the precentral gyrus of BA4, which is the site of the primary motor cortex (i.e., directly related to voluntary movement). This suggests that the interactions depicted in the video trigger higher anticipation of interaction with the webpages.

Third, the area activated specifically for perception of usability is related to task intention [15, 39, 63]. This suggests that perceived usability might implicate some notion of task because assessing usability activates task areas. Some studies relate this area to affordance [34, 62]. Task intention is key to most definitions of usability, for example, ISO 9241, which says that usability is relative to the task. Here, even though many web pages were related to leisure and scored high in pretesting on hedonic quality [37], these areas were still active.

Fourth, Experiment 1 revealed activity in several brain areas related to linguistic processing [61], categorization, and rational thought - in short, higher-order thinking. This could be interpreted in the way that recognizing categories, an essential aspect of navigation, is crucial to assessing usability and could also be distinct for usability. Even without given the opportunity to interact, people still attempt to categorize and recognize navigation options on the webpages. 


\section{Usability and aesthetics of webpages}

From the first experiment, we found some overlap between perceived aesthetic and usability in terms of brain activation. This is in line with previous literature, suggesting that stimuli with high-perceived usability are also highly aesthetic, and vice versa. Our results show that the left medial frontal gyrus (BA10, found in the parametrical effects of aesthetics and familiarity ratings, and aesthetics judgment of pictures) and the inferior parietal lobule (found in response to infant faces versus crosshair baby schema levels) were activated with both perceived usability and aesthetics. This suggests that users might assess some components of perceived usability by substituting an assessment of perceived aesthetics. Likewise, perceived aesthetics may form part of perceived usability, leading to the overlap between these areas.

We found neural correlates of perceived usability during the judgment of static and dynamic stimuli. Although the stimuli during both studies were webpages, which are complex and combine multiple elements, we found that most activation was in areas identified in previous studies of visual aesthetic stimuli (e.g., aesthetics and familiarity ratings [13], faces [72], aesthetics of contour objects [84], baby schema [30]). Our findings confirm that a complex stimulus such as a webpage has its aesthetics judged in the same way as other simpler stimuli.

The results indicate that the right medial frontal gyrus (BA9) is activated during perceived aesthetics judgement, although this has not been investigated previously in the literature on aesthetics. Functionally, the right medial frontal gyrus (BA9) is related to moral judgments (e.g., judging a sentence "right" or "wrong" [65], or probing using moral dilemmas [33]). The anterior cingulate cortex (BA24) was shown to be activated during the process of recognizing error [16]. The activations found in these regions suggest that besides judging whether a webpage is beautiful, participants may have been judging whether this webpage design was right or wrong for the intended purpose. This might have a connection with the task intention aspect of usability indicated earlier.

\section{Implications for future study and limitations}

As our study represents the first attempt to identify the neural correlates of usability, we could not form a strong a priori hypothesis, as is normally done with functional imaging of the brain [23]. There must be a first foundation upon which to propose hypotheses for further investigation - this paper serves that purpose. More in-depth studies of usability can follow our findings to investigate each aspect of usability individually, such as the effects of emotion, anticipation of physical interaction, and task intention.

In addition, the brain activations found in our studies were not just common brain network activations across tasks. There are two reasons for this. First, the brain activations found for perceived aesthetics are in line with the literature on aesthetics, suggesting that they are not just common brain networks. Second, we have analyzed the distinct activations of perceived usability (both as contrast vs. baseline and as differences between levels of usability). Thus, our key results show (a) the activation areas that are distinct from perceived aesthetics and (b) the links between brain area function and the attributes of perceived usability

One limitation of our study is in the experimental protocol. The fact that aesthetics and usability are interrelated presents a challenge; many other applications of fMRI can separate the independent variables much more clearly. One idea used in earlier work is to extensively vary the questions used to ask participants about the webpages. This would have simplified the analysis but would not have allowed us to manipulate usability and aesthetics as we did.

We have not attempted to deal with objective usability, for instance, through task completion times or error rates. Also, we did not treating data as correlational (e.g., correlating ratings and brain activity). Those are obvious directions for future work. However, HCI researchers who employ fMRI technique should consider the difficulty of supporting user interaction in fMRI scanners: participants need to remain static while being scanned. Other limitations include displays with low resolution and the inability to use metal parts in input devices when in a scanner.

\section{Uses for Evaluation and New User Experiences}

One use of our findings is for usability evaluation. We imagine a potential for automated evaluation whereby perceived usability may be assessed over time without asking people, and whereby brain-based evaluations can be used to help distinguish variants of a user interface. Possibly, the most promising use of this would be for assessing highly dynamic stimuli; another idea would be to test the affordance of an interface this way. Currently, fMRI equipment is expensive but portable and low-cost MR scanners are being developed. In addition, we propose dynamically changing user interfaces based on fMRI or a similar technique. This would use adaptive user interfaces that relate to some aspects of usability we have discussed (e.g., anticipation of physical interaction, higher-order thinking) and adapts interfaces based on the scans. Our results could also be used to adapt interfaces on their perceived aesthetics during interaction.

\section{CONCLUSION}

Our paper offers the first exploratory step in investigating usability using a neurological approach. We have used fMRI to identify brain areas specific to usability and areas shared with aesthetics. We have also compared and contrasted perceived usability and aesthetics with the purpose of offering an explanation for why usability and aesthetics are correlated, but are not the same. The dimensions of usability have also been discussed based on an interpretation of the function of brain areas specific to usability, indicating emotion, anticipation of physical interaction, task intentions, and higher-order thinking as key dimensions.

\section{ACKNOWLEDGEMENT}

This work forms part of GHOST, a project funded by the European Commission's 7th Framework Programme, FET- 
Open scheme (Grant \#309191). We thank Dr. Jade Ngoc Thai (CRICBristol, University of Bristol) for the help in providing access to the fMRI scanning facility.

\section{REFERENCES}

1. Ergonomics of human system interaction - Part 210: Human-centred design for interactive systems, in ISO DIS 9241-210. 2008, International Organization for Standardization (ISO).

2. Aharon, I., N. Etcoff, D. Ariely, C.F. Chabris, E. O'Connor, and H.C. Breiter, Beautiful faces have variable reward value: fMRI and behavioral evidence. Neuron, 2001. 32(3): p. 537-51.

3. Al-Shamaileh, O. and A. Sutcliffe, The effect of website interactivity and repeated exposure on user experience, in Proceedings of the 4th Mexican Conference on Human-Computer Interaction. 2012, ACM: Mexico City, Mexico. p. 1-8.

4. Amting, J.M., S.G. Greening, and D.G.V. Mitchell, Multiple Mechanisms of Consciousness: The Neural Correlates of Emotional Awareness. The Journal of Neuroscience, 2010. 30(30): p. 9.

5. Anderson, B.B., C.B. Kirwan, J.L. Jenkins, D. Eargle, S. Howard, and A. Vance, How Polymorphic Warnings Reduce Habituation in the Brain: Insights from an fMRI Study, in CHI. 2015, ACM: Seoul, Republic of Korea. p. 2883-2892.

6. Anderson, E.W., K.C. Potter, L.E. Matzen, J.F. Shepherd, G.A. Preston, and C.T. Silva, A user study of visualization effectiveness using EEG and cognitive load, in EuroVis. 2011, Eurographics Association: Bergen, Norway. p. 791-800.

7. Angeli, A.D., A. Sutcliffe, and J. Hartmann, Interaction, usability and aesthetics: what influences users' preferences?, in Proceedings of the 6th conference on Designing Interactive systems. 2006, ACM: University Park, PA, USA. p. 271-280.

8. Bargas-Avila, J.A. and K. Hornbæk, Old wine in new bottles or novel challenges: a critical analysis of empirical studies of user experience, in CHI. 2011, ACM: Vancouver, BC, Canada. p. 2689-2698.

9. Baumgartner, T., D. Speck, D. Wettstein, O. Masnari, G. Beeli, and L. Jancke, Feeling present in arousing virtual reality worlds: prefrontal brain regions differentially orchestrate presence experience in adults and children. Front Hum Neurosci, 2008. 2: p. 8.

10. Beauregard, M., J. Levesque, and P. Bourgouin, Neural correlates of conscious self-regulation of emotion. $\mathrm{J}$ Neurosci, 2001. 21(18): p. Rc165.

11. Bias, R.G. and D.J. Mayhew, Cost-Justifying Usability: An Update for the Internet Age. 2005: Morgan Kaufmann Publishers Inc.

12. Blakemore, S.-J., D. Bristow, G. Bird, C. Frith, and J. Ward, Somatosensory activations during the observation of touch and a case of vision-touch synaesthesia. Brain, 2005. 128(7): p. 1571-1583.

13. Bohrn, I.C., U. Altmann, O. Lubrich, W. Menninghaus, and A.M. Jacobs, When we like what we know--a parametric fMRI analysis of beauty and familiarity. Brain Lang, 2013. 124(1): p. 1-8.

14. Brainard, D.H., The Psychophysics Toolbox. Spat Vis, 1997. 10(4): p. 433-6.

15. Bush, G., B.A. Vogt, J. Holmes, A.M. Dale, D. Greve, M.A. Jenike, and B.R. Rosen, Dorsal anterior cingulate cortex: A role in reward-based decision making. Proceedings of the National Academy of Sciences, 2002. 99(1): p. 523-528.

16. Carter, C.S., T.S. Braver, D.M. Barch, M.M. Botvinick, D. Noll, and J.D. Cohen, Anterior cingulate cortex, error detection, and the online monitoring of performance. Science, 1998. 280(5364): p. 747-9.

17. Casey, B.J., K.M. Thomas, T.F. Welsh, R.D. Badgaiyan, C.H. Eccard, J.R. Jennings, and E.A. Crone, Dissociation of response conflict, attentional selection, and expectancy with functional magnetic resonance imaging. Proc Natl Acad Sci U S A, 2000. 97(15): p. 8728-33.

18. Cela-Conde, C.J., G. Marty, F. Maestú, T. Ortiz, E. Munar, A. Fernández, M. Roca, J. Rosselló, and F. Quesney, Activation of the prefrontal cortex in the human visual aesthetic perception. PNAS, 2004. 101(16): p. 6321-6325.

19. Chouinard, P.A. and T. Paus, The primary motor and premotor areas of the human cerebral cortex. Neuroscientist, 2006. 12(2): p. 143-52.

20. Cinzia, D.D. and G. Vittorio, Neuroaesthetics: a review. Curr Opin Neurobiol, 2009. 19(6): p. 682-7.

21. Clemente, M., B. Rey, A. Rodríguez-Pujadas, A. BarrosLoscertales, R.M. Baños, C. Botella, M. Alcañiz, and C. Ávila, An fMRI Study to Analyze Neural Correlates of Presence during Virtual Reality Experiences. Interacting with Computers, 2013.

22. CrowdFlower: AI for your business. https://www.crowdflower.com/

23. Dimoka, A., How to conduct a functional magnetic resonance (fMRI) study in social science research. MIS Q., 2012. 36(3): p. 811-840.

24. Ebisch, S.J., M.G. Perrucci, A. Ferretti, C. Del Gratta, G.L. Romani, and V. Gallese, The sense of touch: embodied simulation in a visuotactile mirroring mechanism for observed animate or inanimate touch. $\mathrm{J}$ Cogn Neurosci, 2008. 20(9): p. 1611-23.

25. Elliott, R., K.J. Friston, and R.J. Dolan, Dissociable neural responses in human reward systems. J Neurosci, 2000. 20(16): p. 6159-65. 
26. Foley, E., G. Rippon, N.J. Thai, O. Longe, and C. Senior, Dynamic Facial Expressions Evoke Distinct Activation in the Face Perception Network: A Connectivity Analysis Study. Journal of Cognitive Neuroscience, 2011. 24(2): p. 507-520.

27. Frøkjær, E., M. Hertzum, and K. Hornbæk, Measuring usability: are effectiveness, efficiency, and satisfaction really correlated?, in CHI. 2000, ACM: The Hague, The Netherlands. p. 345-352.

28. Gallagher, H.L., F. Happé, N. Brunswick, P.C. Fletcher, U. Frith, and C.D. Frith, Reading the mind in cartoons and stories: an fMRI study of 'theory of mind' in verbal and nonverbal tasks. Neuropsychologia, 2000. 38(1): p. 11-21.

29. Ge, J., G. Peng, B. Lyu, Y. Wang, Y. Zhuo, Z. Niu, L.H. Tan, A.P. Leff, and J.-H. Gao, Cross-language differences in the brain network subserving intelligible speech. PNAS, 2015. 112(10): p. 2972-2977.

30. Glocker, M.L., D.D. Langleben, K. Ruparel, J.W. Loughead, J.N. Valdez, M.D. Griffin, N. Sachser, and R.C. Gur, Baby schema modulates the brain reward system in nulliparous women. PNAS, 2009. 106(22): p. 9115-9119.

31. Goldberg, I.I., M. Harel, and R. Malach, When the Brain Loses Its Self: Prefrontal Inactivation during Sensorimotor Processing. Neuron, 2006. 50(2): p. 329339.

32. Grecucci, A., C. Giorgetta, N. Bonini, and A.G. Sanfey, Reappraising social emotions: the role of inferior frontal gyrus, temporo-parietal junction and insula in interpersonal emotion regulation. Frontiers in Human Neuroscience, 2013. 7: p. 523.

33. Greene, J.D., R.B. Sommerville, L.E. Nystrom, J.M. Darley, and J.D. Cohen, An fMRI Investigation of Emotional Engagement in Moral Judgment. Science, 2001. 293(5537): p. 2105-2108.

34. Grezes, J., M. Tucker, J. Armony, R. Ellis, and R.E. Passingham, Objects automatically potentiate action: an fMRI study of implicit processing. European Journal of Neuroscience, 2003: p. 6.

35. Hamann, S. and H. Mao, Positive and negative emotional verbal stimuli elicit activity in the left amygdala. Neuroreport, 2002. 13(1): p. 15-9.

36. Hassenzahl, M., The interplay of beauty, goodness, and usability in interactive products. Hum.-Comput. Interact., 2008. 19(4): p. 319-349.

37. Hassenzahl, M., M. Burmester, and F. Koller, AttrakDiff: Ein Fragebogen zur Messung wahrgenommener hedonischer und pragmatischer Qualität, in Mensch \& Computer 2003, G. Szwillus and J. Ziegler, Editors. 2003, Vieweg+Teubner Verlag. p. 187-196.
38. Hassenzahl, M. and N. Tractinsky, User experience-a research agenda. Behaviour \& Information Technology, 2006. 25(2): p. 91-97.

39. Haynes, J.-D., K. Sakai, G. Rees, S. Gilbert, C. Frith, and R.E. Passingham, Reading Hidden Intentions in the Human Brain. Current Biology, 2007. 17(4): p. 323-328.

40. Hertzum, M., Images of Usability. International Journal of Human-Computer Interaction, 2010. 26(6): p. 567600.

41. Hill, A.P. and C.J. Bohil, Applications of Optical Neuroimaging in Usability Research. Ergonomics in design : the magazine of human factors applications, 2016. 24(2): p. 4-9.

42. Hirshfield, L.M., E.T. Solovey, A. Girouard, J. Kebinger, R.J.K. Jacob, A. Sassaroli, and S. Fantini, Brain measurement for usability testing and adaptive interfaces: an example of uncovering syntactic workload with functional near infrared spectroscopy, in ACM CHI Conference on Human Factors in Computing Systems. 2009, ACM: Boston, MA, USA. p. 2185-2194.

43. Hornbæk, K., Current practice in measuring usability: Challenges to usability studies and research. International Journal of Human-Computer Studies, 2006. 64(2): p. 79-102.

44. Huettel, S.A., A.W. Song, and G. McCarthy, Functional Magnetic Resonance Imaging. 2009: Sinauer Associates, Incorporated.

45. Jacobs, R.H.A.H., R. Renken, and F.W. Cornelissen, Neural Correlates of Visual Aesthetics - Beauty as the Coalescence of Stimulus and Internal State. PLoS ONE, 2012. 7(2): p. e31248.

46. Jacobsen, T., R.I. Schubotz, L. Hofel, and D.Y. Cramon, Brain correlates of aesthetic judgment of beauty. Neuroimage, 2006. 29(1): p. 276-85.

47. Kampe, K.K., C.D. Frith, R.J. Dolan, and U. Frith, Reward value of attractiveness and gaze. Nature, 2001. 413(6856): p. 589.

48. Karapanos, E., User Experience Over Time, in Modeling Users' Experiences with Interactive Systems. 2013, Springer Berlin Heidelberg: Berlin, Heidelberg. p. 5783.

49. Kawabata, H. and S. Zeki, Neural correlates of beauty. J Neurophysiol, 2004. 91(4): p. 1699-705.

50. Kim, H., R. Adolphs, J.P. O'Doherty, and S. Shimojo, Temporal isolation of neural processes underlying face preference decisions. PNAS, 2007. 104(46): p. 1825318258.

51. Kühn, S. and J. Gallinat, The neural correlates of subjective pleasantness. NeuroImage, 2012. 61(1): p. 289-294.

52. Kurth, R., K. Villringer, G. Curio, K.J. Wolf, T. Krause, J. Repenthin, J. Schwiemann, M. Deuchert, and A. 
Villringer, fMRI shows multiple somatotopic digit representations in human primary somatosensory cortex. Neuroreport, 2000. 11(7): p. 1487-91.

53. Landauer, T.K., The Trouble with Computers: Usefulness, Usability, and Productivity. 1996: MIT Press.

54. Laugwitz, B., T. Held, and M. Schrepp, Construction and Evaluation of a User Experience Questionnaire, in $\mathrm{HCI}$ and Usability for Education and Work, A. Holzinger, Editor. 2008, Springer Berlin Heidelberg. p. 63-76.

55. Law, E.L.-C., V. Roto, M. Hassenzahl, A.P.O.S. Vermeeren, and J. Kort, Understanding, scoping and defining user experience: a survey approach, in CHI. 2009, ACM: Boston, MA, USA. p. 719-728.

56. Lewis, J.R., Usability: Lessons Learned ... and Yet to Be Learned. International Journal of Human-Computer Interaction, 2014. 30(9): p. 663-684.

57. Lindgaard, G. and C. Dudek, What is this evasive beast we call user satisfaction? Interacting with Computers, 2003. 15(3): p. 429-452.

58. Lindgaard, G., C. Dudek, D. Sen, L. Sumegi, and P. Noonan, An exploration of relations between visual appeal, trustworthiness and perceived usability of homepages. ACM Trans. Comput.-Hum. Interact., 2011. 18(1): p. 1-30.

59. Lindgaard, G., G. Fernandes, C. Dudek, and J. Brown, Attention web designers: you have 50 milliseconds to make a good first impression!

60. Lukanov, K., H.A. Maior, and M.L. Wilson, Using fNIRS in Usability Testing: Understanding the Effect of Web Form Layout on Mental Workload, in Proceedings of the 2016 CHI Conference on Human Factors in Computing Systems. 2016, ACM: Santa Clara, California, USA. p. 4011-4016.

61. McCandliss, B.D., L. Cohen, and S. Dehaene, The visual word form area: expertise for reading in the fusiform gyrus. Trends in Cognitive Sciences, 2003. 7(7): p. 293299.

62. Mecklinger, A., C. Gruenewald, N. Weiskopf, and C.F. Doeller, Motor affordance and its role for visual working memory: evidence from fMRI studies. Exp Psychol, 2004. 51(4): p. 258-69.

63. Miller, E.K., D.J. Freedman, and J.D. Wallis, The prefrontal cortex: categories, concepts and cognition. Philosophical Transactions of the Royal Society B: Biological Sciences, 2002. 357(1424): p. 1123-1136.

64. Molenberghs, P., R. Cunnington, and J.B. Mattingley, Is the mirror neuron system involved in imitation? A short review and meta-analysis. Neuroscience \& Biobehavioral Reviews, 2009. 33(7): p. 975-980.
65. Moll, J., P.J. Eslinger, and R. Oliveira-Souza, Frontopolar and anterior temporal cortex activation in a moral judgment task: preliminary functional MRI results in normal subjects. Arq Neuropsiquiatr, 2001. 59(3-b): p. 657-64.

66. Monti, M.M., L.M. Parsons, and D.N. Osherson, The boundaries of language and thought in deductive inference. PNAS, 2009. 106(30): p. 12554-12559.

67. Moshagen, M. and M.T. Thielsch, Facets of visual aesthetics. International Journal of Human-Computer Studies, 2010. 68(10): p. 689-709.

68. Nielsen, J., Usability Engineering. 1994: AP Professional.

69. Paulus, M.P. and L.R. Frank, Ventromedial prefrontal cortex activation is critical for preference judgments. Neuroreport, 2003. 14(10): p. 1311-5.

70. Pedersen, E.W., S. Subramanian, and K. Hornbæk. Is my Phone Alive? A Large-Scale Study of Shape Change in Handheld Devices Using Videos. CHI. 2014. ACM.

71. Peirce, C.S. and J. Buchler, Philosophical Writings of Peirce. 2012: Dover Publications.

72. Phillips, M.L., L. Williams, C. Senior, E.T. Bullmore, M.J. Brammer, C. Andrew, S.C.R. Williams, and A.S. David, A differential neural response to threatening and non-threatening negative facial expressions in paranoid and non-paranoid schizophrenics. Psychiatry Research: Neuroimaging, 1999. 92(1): p. 11-31.

73. Pine, D.S., J. Grun, E.A. Maguire, N. Burgess, E. Zarahn, V. Koda, A. Fyer, P.R. Szeszko, and R.M. Bilder, Neurodevelopmental aspects of spatial navigation: a virtual reality fMRI study. Neuroimage, 2002. 15(2): p. 396-406.

74. Pochon, J.B., R. Levy, P. Fossati, S. Lehericy, J.B. Poline, B. Pillon, D. Le Bihan, and B. Dubois, The neural system that bridges reward and cognition in humans: An fMRI study. PNAS, 2002. 99(8): p. 56695674.

75. Poldrack, R.A., Can cognitive processes be inferred from neuroimaging data? Trends Cogn Sci, 2006. 10(2): p. 59-63.

76. Pólya, G., Mathematics and Plausible Reasoning: Induction and analogy in mathematics. 1990: Princeton University Press.

77. Purves, D., Neuroscience. 2012: Sinauer Associates.

78. Rizzolatti, G. and L. Craighero, The mirror-neuron system. Annu Rev Neurosci, 2004. 27: p. 169-92.

79. Sauro, J. and J.R. Lewis, Correlations among prototypical usability metrics: evidence for the construct of usability, in CHI. 2009, ACM: Boston, MA, USA. p. 1609-1618.

80. Sjölie, D., K. Bodin, E. Elgh, J. Eriksson, L.-E. Janlert, and L. Nyberg, Effects of interactivity and 3D-motion 
on mental rotation brain activity in an immersive virtual environment, in CHI. 2010, ACM: Atlanta, Georgia, USA. p. 869-878.

81. Tractinsky, N., A. Cokhavi, M. Kirschenbaum, and T. Sharfi, Evaluating the consistency of immediate aesthetic perceptions of web pages. IJHCS, 2006. 64(11): p. 1071-1083.

82. Tuch, A.N., S.P. Roth, K. Hornbæk, K. Opwis, and J.A. Bargas-Avila, Is beautiful really usable? Toward understanding the relation between usability, aesthetics, and affect in HCI. Computers in Human Behavior, 2012. 28(5): p. 1596-1607.

83. Vartanian, O. and V. Goel, Neuroanatomical correlates of aesthetic preference for paintings. Neuroreport, 2004. 15(5): p. 893-7.
84. Vartanian, O., G. Navarrete, A. Chatterjee, L.B. Fich, H. Leder, C. Modroño, M. Nadal, N. Rostrup, and M. Skov, Impact of contour on aesthetic judgments and approachavoidance decisions in architecture. PNAS, 2013. 110(Supplement 2): p. 10446-10453.

85. Vi, C., K. Takashima, H. Yokoyama, G. Liu, Y. Itoh, S. Subramanian, and Y. Kitamura, D-FLIP: Dynamic and Flexible Interactive PhotoShow, in Advances in Computer Entertainment, D. Reidsma, H. Katayose, and A. Nijholt, Editors. 2013, Springer. p. 415-427.

86. Yamamoto, S., I.E. Monosov, M. Yasuda, and O. Hikosaka, What and where information in the caudate tail guides saccades to visual objects. J Neurosci, 2012. 32(32): p. 11005-16. 